\title{
COMPARISION BETWEEN ULTRASOUND GUIDED SERRATUS ANTERIOR PLANE BLOCK AND THORACIC EPIDURAL FOR POSTOPERATIVE
}

ANALGESIA AFTER MODIFIED RADICAL MASTECTOMY

Sherif Younis Amin Omar, Mounir Kamal Afify, Mohamed Mahmoud Abd El-Hady, Mohamed Abd El-HadyAbd El-Hameed

Department of Anaesthesia and Surgical Intensive Care, Faculty of Medicine University of Alexandria

\section{INTRODUCTION}

Patients undergoing breast surgery require a multimodal postoperative pain treatment regimen that provides high quality analgesia with minimal side effects.

Thoracic Epidural Analgesia and paravertebral blocks became the gold standard to achieve postoperative pain relief after breast surgery, but not every anaesthiologist is comfortable performing these procedures. As an alternative for these technigues Serratus anterior plane block was designed.

SAPB involves depositing LA in the plane superficial or deep to the serratus anterior muscle, and has been suggested to provide analgesia in similar surgeries involving the breast and hemithorax.

\section{AIM OF THE WORK}

The primary aim of this study was to compare between the efficiency of serratus anterior plane block and thoracic epidural analgesia in terms of postoperative pain anterior plane block and thoracic epidural analgesia
relief in the 1 st 24 hours determined by VAS score.

\section{PATIENTS AND METHODS}

PATIENTS: 40 female patients aged 25-50 years old.

Exclusion criteria:

- History of allergy or contraindication to any of the studied drugs.

- Patient refusal.

- Skin infection at the site of injection.

Bleeding disorders or receiving anticoagulant.

- Spine or chest wall deformity.

- Body mass index more than $35 \mathrm{~kg} / \mathrm{m}^{2}$.

METHODS:

Patient's preparation:

- Full medical, surgical, anaesthetic history will be taken from every patient.

- Physical examination and evaluation of the airway will be done.

- Routine laboratory investigations will be reviewed.

- All patients will be taught how to interpret the visual analogue scale (VAS) of 0 to $10 \mathrm{~cm}$ with 0 being no pain and $10 \mathrm{~cm}$ being the worst pain imaginable.

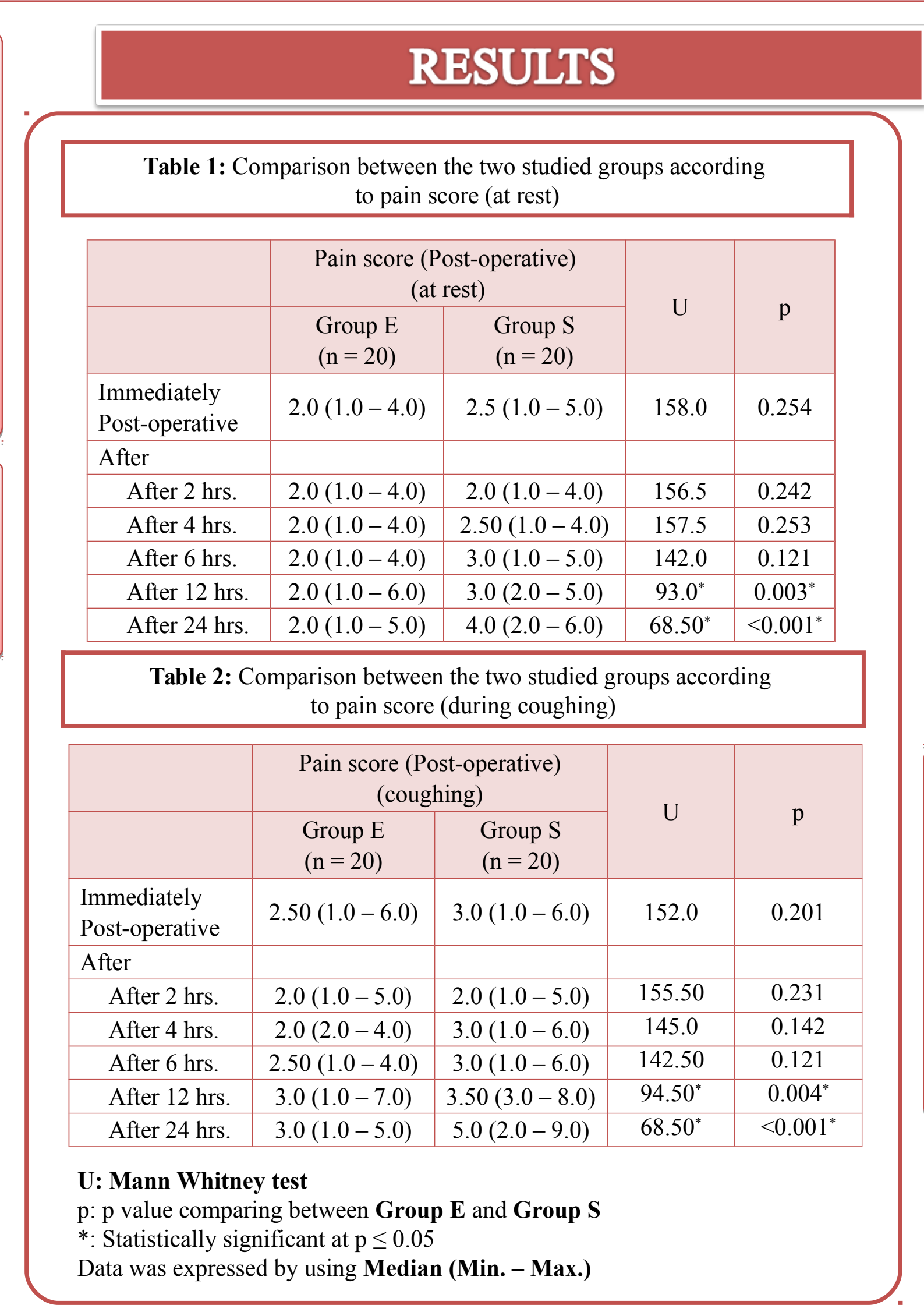

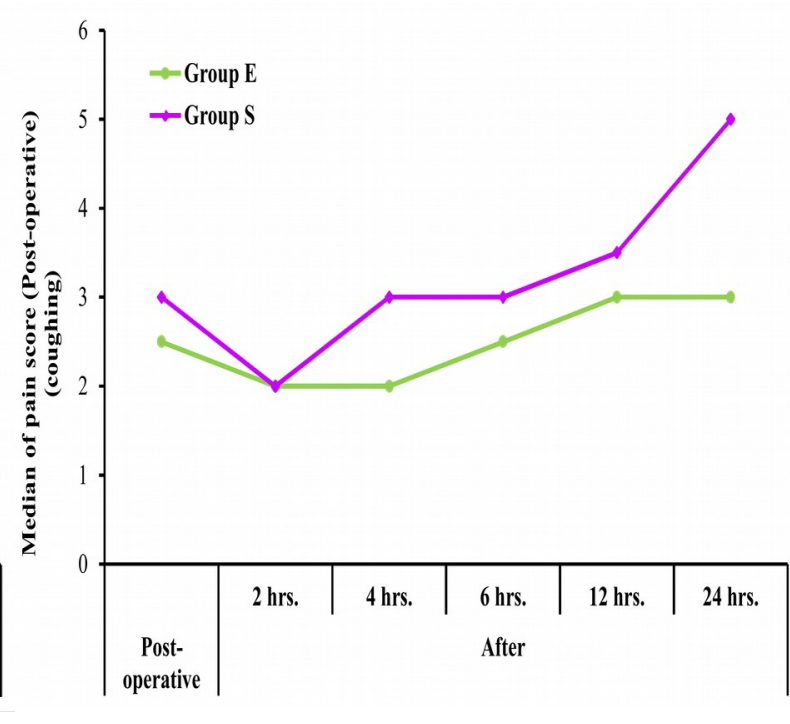

Figure 1: Comparison between the two studied groups according to pain score Figure 2: Comparison between the two studied groups according to pain score (during coughing)

\section{CONCLUSION}

-The time taken for performing Serratus Anterior Plan Block was significantly shorter than time take to insert Thoracic epidural catheter.

and safe technique thoracic epidural analgesia with comparable post-operative VAS score in the $1^{\text {st }} 24$ hours.

-The total post-operative rescue analgesia was more in the SAPB group than TEA group.

-Analgesia duration in SAPB group was shorter than in TEA group.

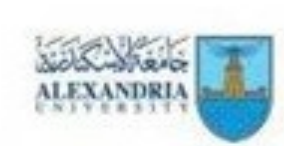$$
\text { 2021@Alexandria Faculty of Medicine }
$$

MEDICINE | 